Received: 2018.11.17 Accepted: 2019.04.22 Published: 2019.08.23
Authors' Contribution:

Study Design A

Data Collection B

Statistical Analysis C

Data Interpretation D

Manuscript Preparation E

Literature Search F

Funds Collection G
Alaa A. Alhubaishi

\title{
Pancytopenia and Septic Infection Caused by Concurrent Use of Allopurinol and Mercaptopurine: A Case Report Illustrating the Importance of Clinical Pharmacist Consultation
}

Department of Pharmacy Practice, Collage of Pharmacy, Princess Nora University, Riyadh, Saudi Arabia
Corresponding Author: Conflict of interest:
Alaa A. Alhubaishi, e-mail: aaalhubaishi@pnu.edu.sa

None declared

\begin{abstract}
Patient: Female, 55
Final Diagnosis:

Symptoms:

Medication:

Clinical Procedure:

Specialty:

Objective:

Background:

Case Report:

Conclusions:

Allopurinol and mercaptopurine interaction causing pancytopenia and septic infection

Sepsis

Fluid transfusion and cephalexin

Pharmacology and Pharmacy

\section{Adverse events of drug therapy}

Pancytopenia is a hematological condition which is characterized by decreases in all three cellular elements: RBC, WBC, and platelets. As a result, patients with pancytopenia are more prone to anemia, infections, and excessive bleeding. Pancytopenia can be caused by medications or drug interactions that suppress the bone marrow. This case report highlights a drug interaction between allopurinol and mercaptopurine which led to pancytopenia and septic infection, resulting in the patient's death. This could easily have been avoided if a clinical pharmacist had been consulted.

A 55-year-old female patient with a past medical history of gout, depression, back pain, and type 2 diabetes was recently diagnosed with ulcerative colitis and was discharged with a new prescription of mercaptopurine. After 2 months of concurrent use of allopurinol and mercaptopurine, she developed infected foot ulcers, which progressed rabidly to sepsis. At the time, her laboratory findings confirmed pancytopenia. Despite treatment, the patient died.

This case illustrates the importance of consulting a clinical pharmacist in order to avoid such medical error. The dose of mercaptopurine should be reduced to $25 \%$ of the recommended dose when it is given concurrently with allopurinol to reduce the risk of pancytopenia. Health care providers should think about the significant role of clinical pharmacy services. In our case, there were no clinical pharmacist involved in the care of this patient, and as a result of such negligence, the patient lost her life.
\end{abstract}

MeSH Keywords:

Full-text PDF:

Drug Interactions • 6-Mercaptopurine • Pharmacists • Allopurinol • Pancytopenia

https://www.amjcaserep.com/abstract/index/idArt/914166

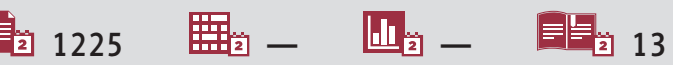




\section{Background}

Pancytopenia is a hematological condition which is characterized by decreases in all three cellular elements: RBC, WBC, and platelets [1]. It is defined as hemoglobin less than $13.5 \mathrm{~g} / \mathrm{dl}$ in males or $11.5 \mathrm{~g} / \mathrm{dl}$ in females, leucocyte count less than $4.5 \times 10^{9} / \mathrm{L}$, and platelet count less than $15 \times 10^{\%} / \mathrm{L}$ [2]. Pancytopenia can be life threating and may evolve insidiously in some cases. The causes of pancytopenia vary widely in different studies [3,4]. Megaloblastic anemia is considered the most common cause of pancytopenia; however, there are other underlying causes. Medications or drug interactions that suppress the bone marrow have been also reported to cause pancytopenia $[5,6]$. Allopurinol is a xanthine oxidase inhibitor indicated for management of patients with gout. The most frequent adverse reaction to allopurinol is skin rash [7]. Mercaptopurine is a thiopurine which is used for a number of conditions related to immune diseases. It is also used off label for inflammatory bowel diseases, including Crohn's disease and ulcerative colitis. Serious toxic effects of mercaptopurine include myelosuppression and hepatotoxicity [8]. There are few published case reports describing pancytopenia as a result of concurrent use of allopurinol and thiopurines. The published case reports are likely to represent only a small fraction of the actual cases because pancytopenia may not be recognized as being the result of drug interactions [5,9]. This interaction is very old, and it was first described in 1963 by Gertrude Elion, who reported that giving a xanthine oxidase inhibitor with a thiopurine can substantially increase the effect of the thiopurine, leading to bone marrow suppression, and that will reduce the activation and replication of blood cells, inducing apoptosis and causing pancytopenia [10]. Therefore, the dose of thiopurine should be reduced to $25 \%$ of the recommended dose when allopurinol is given concurrently. In contrast, other studies reported the intentional use of allopurinol with mercaptopurine in some inflammatory bowel disease cases to enhance thiopurine treatment; however, the dose of mercaptopurine was managed [11,12]. Here we report the case of a 55-year-old woman who died after a septic infection in the presence of pancytopenia after taking mercaptopurine while on allopurinol. This incident occurred in the absence of clinical pharmacy services in one of the hospitals in the Riyadh region of Saudi Arabia.

\section{Case Report}

A 55-year-old female patient with a history of gout, depression, back pain, and type 2 diabetes was recently diagnosed with ulcerative colitis. A new prescription of mercaptopurine $100 \mathrm{mg}$ daily was dispensed and she was told to continue all other medications. She was taking allopurinol $300 \mathrm{mg}$ twice daily, fluoxetine $150 \mathrm{mg}$ daily, ibuprofen $200 \mathrm{mg}$ twice daily as needed, metformin $500 \mathrm{mg}$ twice daily, and glipizide $300 \mathrm{mg}$ twice daily. Two months prior to her admission, she had received allopurinol and mercaptopurine concurrently. During that time, a progressive pancytopenia had developed to the point that, on admission, her hemoglobin level was $8.6 \mathrm{~g} / \mathrm{dL}$, WBC count was $2.2 \times 10^{9} / \mathrm{L}$, and platelet count was $9.35 \times 10^{9} / \mathrm{L}$. Bone marrow aspiration excluded hypoplastic anemia hematological malignancies, megaloblastic anemia, leishmaniasis, and Gaucher disease. At this point, the pancytopenia etiology was not recognized.

On admission, the patient was confused, anxious, and pale. She complained of breathlessness and palpitation. The patient was febrile, with body temperature of $39.6^{\circ} \mathrm{C}$, heart rate of 112 beat/min, respiratory rate of 26 breaths/min, $\mathrm{SaO} 288 \%$, and BP $80 / 68 \mathrm{mmHg}$. She was hemodynamically unstable at the time of admission. One liter of isotonic solution was transfused immediately. The laboratory investigation showed sodium $138 \mathrm{mmol} / \mathrm{L}$, potassium $5.5 \mathrm{mmol} / \mathrm{L}$, chloride $120 \mathrm{mmol} / \mathrm{L}$, bicarbonate $12 \mathrm{mmol} / \mathrm{L}$, BUN $30 \mathrm{mg} / \mathrm{dL}$, creatinine $2.9 \mathrm{mg} / \mathrm{dL}$, uric acid $4 \mathrm{mg} / \mathrm{dL}$, glucose $490 \mathrm{mg} / \mathrm{dL}$, A 1c $11.2 \%$, albumin $22 \mathrm{~g} / \mathrm{L}$, AST $56 \mathrm{U \backslash L}$, ALT $60 \mathrm{U} / \mathrm{L}$, neutrophils $0.9 \times 10^{9} / \mathrm{L}$, and serum lactate $7 \mathrm{mmol} / \mathrm{L}$.

Patient's both feet were covered with very moist and foul-smelling gauzes. Her family stated that she had stepped on something sharp 5 days ago and had developed bloody ulcers, but she did not seek medical care at that time. On physical examination, her ulcers were reddish and edematous; a yellowish discharge was noted from her ulcerated skin lesions. It was primarily diagnosed as diabetic foot ulcer. Blood and wound cultures were obtained and the patient was given cephalexin intravenously. After the initial fluid transfusion, no improvement was observed in patient status. Within 1 hour of admission, sepsis developed and the patient died.

\section{Discussion}

It is known that pancytopenia can result from concurrent use of allopurinol and mercaptopurine. Patients with pancytopenia are fatigued due to anemia, more susceptible to infections due to leucopenia, and are at risk of excessive bleeding due to thrombocytopenia. There are very few case reports in the literature regarding pancytopenia risk as a result of xanthan oxidase inhibitor and thiopurine interaction. In 1970, it was reported that a 61-year-old man on azathioprine died of pancytopenia, bleeding, and sepsis after starting allopurinol therapy [5]. In 2009, the New Zealand Health Department issued a safety notice after a patient on azathioprine and allopurinol died of pancytopenia [9].

In our case, the patient was concurrently on allopurinol $300 \mathrm{mg}$ twice daily and mercaptopurine $100 \mathrm{mg}$ daily for 2 months 
prior to admission. During that time, she had gradually developed pancytopenia, which had increased her risk of infections.

Her foot ulcers developed from an injury 5 days prior to her admission. At that time, her immune system was not functioning well enough to fight her infection, not only because of pancytopenia, but also due to her history of uncontrolled diabetes. High blood glucose levels can weaken the immune system defenses [13]. Moreover, myelosuppression is known as a side effect of mercaptopurine, and the concurrent use with allopurinol definitely increases the risk of myelosuppression, resulting in pancytopenia with the exclusion of other hematological causes. Because of the pancytopenia, foot infection, and hemodynamic instability, the possibility of bacterial sepsis was strongly considered. A blood culture showed grampositive cocci later identified as Staphylococcus aureus. We concur with other authors that predisposition to infection or sepsis in patients with pancytopenia can be induced by an interaction between allopurinol and a thiopurine. The evaluation of this patient's medication history and the possibility of drug interaction with the new prescription was missed in this case. Failing to recognize or monitor for this interaction increased the risk of pancytopenia and infections. The interaction between allopurinol and mercaptopurine certainly could have been avoided if the mercaptopurine dose was reduced as recommended and the patient was monitored. This is an example of the importance of the involvement of a clinical pharmacist in patient's care to avoid such tragedy.

\section{References:}

1. Gayathri BN, Rao KS: Pancytopenia: A clinico-hematological study. J Lab Physicians, 2011; 3(1): 15-20

2. Naseem S, Varma N, Das R et al: Pediatric patients with bicytopenia/pancytopenia: Review of etiologies and clinico-hematological profile at a tertiary center. Indian J Pathol Microbiol, 2011; 54: 75-80

3. Das Makheja K, Kumar Maheshwari B, Arain S et al: The common causes leading to pancytopenia in patients presenting to tertiary care hospital. Pak J Med Sci, 2013; 29(5): 1108-11

4. Imbert M, Scoazec JY, Mary JY et al: Adult patients presenting with pancytopenia: A reappraisal of underlying pathology and diagnostic procedures in 213 cases. Hematol Pathol, 1989; 3: 159-67

5. Gearry RB, Day AS, Barclay ML et al: Azathioprine and allopurinol: A twoedged interaction. J Castroenterol Hepatol, 2010; 25: 649-56

6. NSW Health Safety Notice. Allopurinol and Azathioprine. A Serious and Known Drug Interaction, 2009; 011/09: 1-2. Available from URL: http:// www.health.nsw.gov.au/resources/quality/sabs/pdf/sn20090507.pdf

\section{Conclusions}

Most hospitals in Saudi Arabia lack clinical pharmacy services. This case illustrates a significant medical error which resulted in a patient's death. The drug interaction between allopurinol and mercaptopurine contributed to pancytopenia, which reduced the patient's ability to fight her infection, resulting in sepsis and death. It is recommended to reduce the dose of mercaptopurine to $25 \%$ of the recommended dose when combined with allopurinol. There is a significant need for a qualified clinical pharmacist to be included in the health care team with other professions to perform their role as drug experts. In our case, there were no clinical pharmacist involvement in the care of this patient; as the result of such negligence, the patient lost her life.

\section{Acknowledgements}

The author would like to thank Dr. Tarq F Alkhowaiter for his kind support and assistance in writing.

\section{Conflict of interests}

None.

7. Allopurinol. Lexi-Drugs.Lexicomp. Wolters Kluwer Health, Inc. Riverwood IL. Available at: http://online.lexi..com

8. Mercaptopurine. Lexi-Drugs.Lexicomp. Wolters Kluwer Health, Inc. Riverwood, IL. Available at: $h$ ttp://online.lexi..com

9. Horn JR, Hansten PH: Another thiopurine- allopurinol tragedy. Retrieved from http://www.hanstenandhorn.com/news.htm

10. Mclnnes GT, Lawson DH, Jick H: Acute adverse reactions attributed to allopurinol in hospitalised patients. Ann Rheum Dis,1981; 40(3): 245-49

11. DeConti RC, Calabresi P: Use of Allopurinol for prevention and control of hyperuricemia in patients with neoplastic disease. New Engl J Med, 1966; 274: 481-86

12. Moreau B, Clement $P$, Theoret $Y$, Seidman EG: Allopurinol in combination with thiopurine induces mucosal healing and improves clinical and metabolic outcomes in IBD. Therap Adv Gastroenterol, 2017; 10(11): 819-27

13. Casqueiro J, Casqueiro J, Alves C: Infections in patients with diabetes mellitus: A review of pathogenesis. Indian J Endocrinol Metab, 2012; 16(Suppl. 1): $S 27-36$ 